\title{
Les domaines d'application du Jet grouting
}

\author{
Jet grouting in construction
}

\section{J. MOREY}

$\mathrm{BACHY}^{*}$

Rev. Franç. Géotech. n 61, pp. 17-30 (décembre 1992)

\section{Résumé}

Différentes méthodes de jet grouting (jet simple, jet double, jet triple) sont utilisées en fonction de l'énergie hydrodynamique nécessaire pour détruire et mélanger le terrain avec un coulis liquide. Elles s'appliquent aussi bien à des traitements de masse que des traitements linéaires ou des inclusions, dans des sols meubles facilement ou difficilement injectables. Les problèmes d'utilisation concernent la définition géométrique et mécanique du résultat obtenu et son contrôle, ainsi que les difficultés d'évacuation des matériaux liquides en excès formés pendant la production.

\section{Abstract}

Different methods of jet grouting (single, double, triple jet) are used in construction, depending on the hydrodynamic energy required to dessaggregate and mix the ground with grout. They are used for mass treatment as well as to form jet grout curtains or to obtain isolated columns, and this in various soils, independantly from their groutability with the conventional grouting techniques. Main concerns about the jet grouting process are the geometrical and mechanical definition and control of the end products, and the difficulties on site for the evacuation of the spoiling materials in excess generated during production. 


\section{RAPPEL DES PRINCIPES DU JET GROUTING}

On désigne par « jet grouting un procédé de construction utilisant un jet de fluide à haute énergie cinétique pour déstructurer un terrain et le mélanger avec un coulis liquide.

Il ne s'agit donc pas exactement d'une technique d'injection, mais plutôt d'un procédé de mélange hydrodynamique terrain-coulis visant à former un "béton de sol » in situ dans la masse du terrain. C'est pourquoi on préfère employer la dénomination a jetmix », laquelle qualifie mieux les phénomènes physiques impliqués et le rôle du terrain constituant partiellement le mélange final.

L'idée du jetmix est née au Royaume Uni à la fin des années 50 . Une première réalisation au Pakistan, bien que couronnée de succès, n'eut pas de suite. Il fallut attendre le début des années 70 pour voir lidée reprise au Japon, avec des matériels différents. Depuis, les procédés se sont multipliés, et leur domaine d'application dans la construction n'a cessé de s'étendre.

Le jet grouting fait appel, séparément ou en combinaison, à trois phénomènes physiques :

- déstructuration du terrain par un jet à très grande vitesse ;

- extraction d'une partie du terrain jusqu'à la surface par les fluides de jetting ;

- incorporation d'un liant apporté par le coulis.

Le traitement se déroule généralement ainsi (fig. 1) : - réalisation d'un forage de petit diamètre (100 à $200 \mathrm{~mm}$ ) sur la hauteur à traiter ;

- dans ce forage, mise en vitesse d'un jet de fluide envoyé par une pompe à haute pression (plusieurs dizaines de $\mathrm{MPa}$ ) à travers une ou plusieurs buses de petit diamètre (1 à $10 \mathrm{~mm}$ ) placées sur un « moniteur a au pied d'un train de tiges de diamètre 70 à $100 \mathrm{~mm}$
- remontée lente des tiges, avec mise en rotation pour former une colonne de béton de sol avec le coulis envoyé sur la hauteur traitée depuis le pied du train de tiges.

Pendant la phase jet, les volumes en excès du mélange sol-ciment (appelés « spoil ») doivent ressortir librement en tête de forage (sinon, l'excès de matériaux risque de "claquer * le terrain et de créer des désordres au voisinage) et être évacués du chantier au fur et à mesure.

Le résultat (diamètre, composition, résistance des colonnes) dépend des paramètres du traitement (vitesses de translation et de rotation des tiges, pression et débit des fluides utilisés, dosage du coulis), des caractéristiques du terrain en place (nature, granulométrie, composition, compacité) et de la méthode employée (simple, double ou triple).

\section{LES PRINCIPALES MÉTHODES}

\subsection{Jet simple (fig. 2)}

Dans ce procédé, le fluide du jet destructeur est le coulis lui-même, qui assure les trois fonctions de base de déstructuration, extraction et incorporation.

Une partie importante de l'énergie cinétique du jet est dissipée par frottement dans le terrain mis en suspension et le spoil formé devient parfois trop visqueux pour remonter librement jusqu'en tête de forage.

\subsection{Jet double (fig. 2)}

Si on protège le jet de coulis à très grande vitesse par un jet d'air annulaire, le rayon d'action du jet destructeur en face du même terrain se trouve considérablement augmenté par le cône d'air enveloppant.

Dans le procédé jet double, le rôle de l'air est aussi d'améliorer les possibilités d'extraction du terrain en place par phénomène d'air lift.
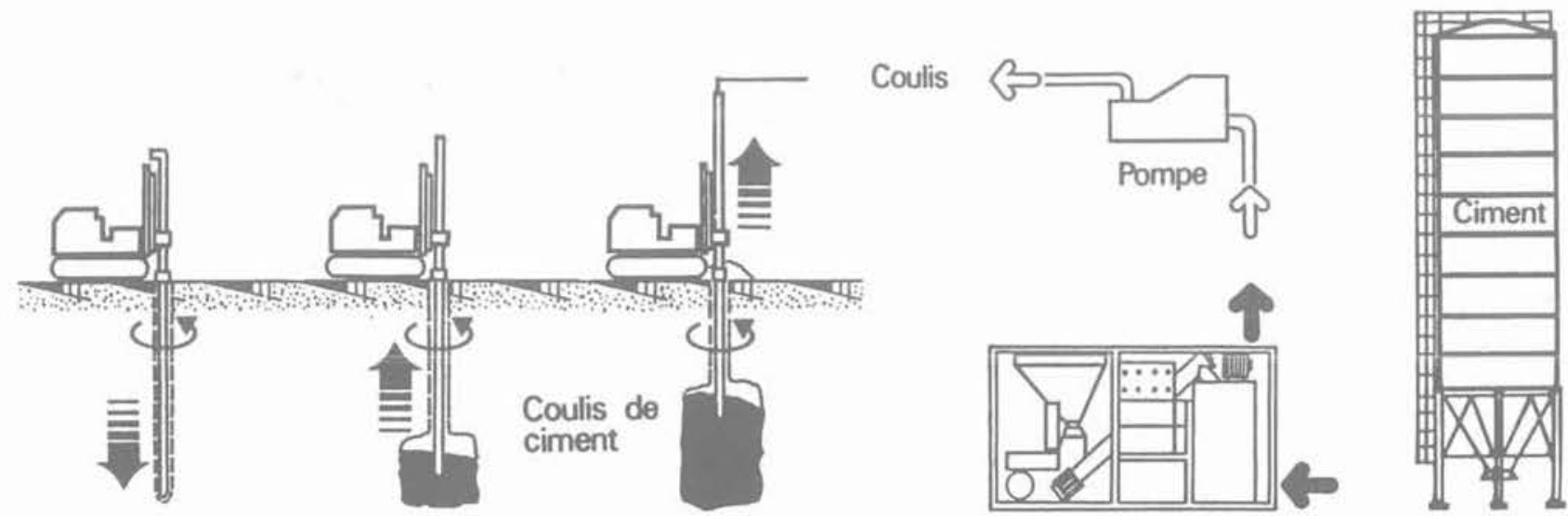

Fig. 1. - Jet Mix, réalisation de colonnes.

Fig. 1. - Jet Mix, columns construction. 


\section{JET UNIQUE}

(ciment)

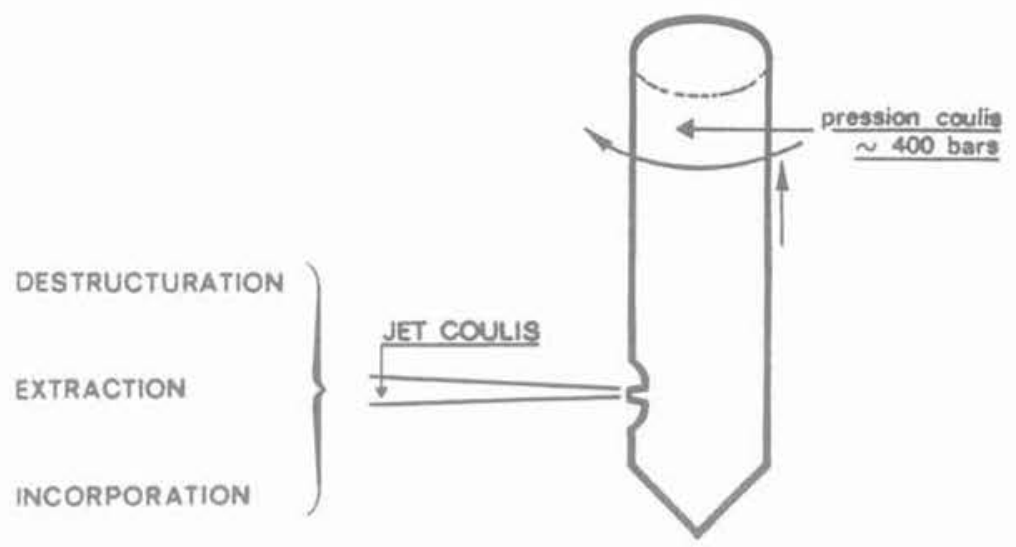

\section{JET DOUBLE}

(ciment+air)
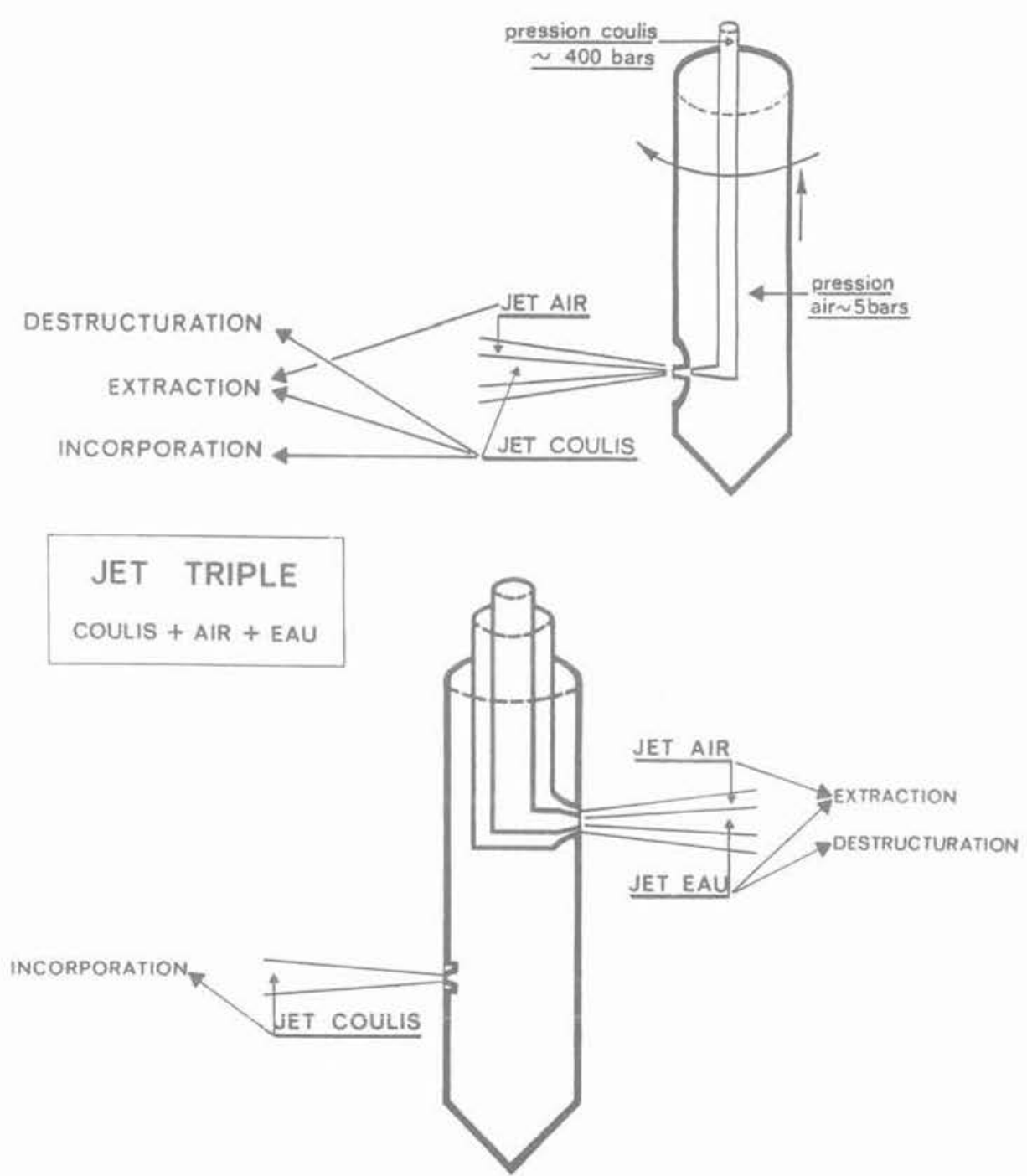

Fig. 2. - Principales méthodes.

Fig. 2. - Main methods 


\subsection{Jet triple (fig. 2)}

Dans le jet triple, les fonctions déstructuration et extraction du terrain sont obtenues par un jet double d'eau et d'air séparément de la fonction d'incorporation du liant assurée par un jet de coulis envoyé simultanément à basse pression (quelques $\mathrm{MPa}$ ), généralement par une buse inférieure.

La pompe et le circuit haute pression utilisés en jet triple ne supportent que de l'eau, avec en contre partie la nécessité d'une pompe supplémentaire à basse pression pour le coulis et d'une ligne d'outils à triple tube.

\subsection{Comparaison des méthodes}

La comparaison la plus significative concerne les diamètres de colonnes que l'on obtient généralement par le procédé jet simple et par les procédés avec jet entouré d'air (jet double, jet triple) en face des terrains les plus courants (fig. 3).

Bien entendu, les termes de la comparaison dépendent des paramètres employés pour le traitement, mais on constate que le rayon d'action double à énergie égale entre jet simple et jet double ou jet triple. Il est ainsi exceptionnel d'arriver à obtenir économiquement des diamètres approchant $1 \mathrm{~m}$ avec le jet simple, alors que les procédés avec air peuvent per- mettre de dépasser aisément $1 \mathrm{~m}$ et d'atteindre même 2 à $3 \mathrm{~m}$ dans certaines circonstances.

Evidemment, ces performances dépendent du terrain et plus précisément de :

- la granulométrie du sol (plus le terrain est fin, plus il est difficile à déstructurer) ;

- la compacité du sol (plus le terrain est cohérent ou dense, moins le jet est efficace).

\subsection{Méthodes dérivées}

Les dénominations jet simple, jet double, jet triple décrivent le procédé de jetting, indépendamment du nombre et de la position des buses et donc des jets utilisés (par exemple, on emploie fréquemment deux buses diamétralement opposées en jet simple), sans plus de précisions quand on réalise des colonnes en une seule phase de jetting.

Mais ces mêmes procédés sont également employés dans des méthodes dérivées telles que :

- le panel jet (fig. 4) : le moniteur est remonté sans rotation, formant un panneau de jetmix dont l'épaisseur varie de 5 à $30 \mathrm{~cm}$ selon les terrains et le procédé (dans les terrains fins et compacts, la coupure est plus mince que dans les sables et graviers), sauf si des éléments durs grossiers (de taille supérieure à quelques $\mathrm{cm}$ ) s'opposent au passage du jet, auquel cas la forme en panneau n'est pas garantie ;

\section{COMPARAISON JET SIMPLE (1) / JET TRIPLE (2)}

\section{LIMON ARGILEUX}

SABLE LIMONEUX

SABLE ET GRAVIER

\section{ALLUVIONS GROSSIÈRES}

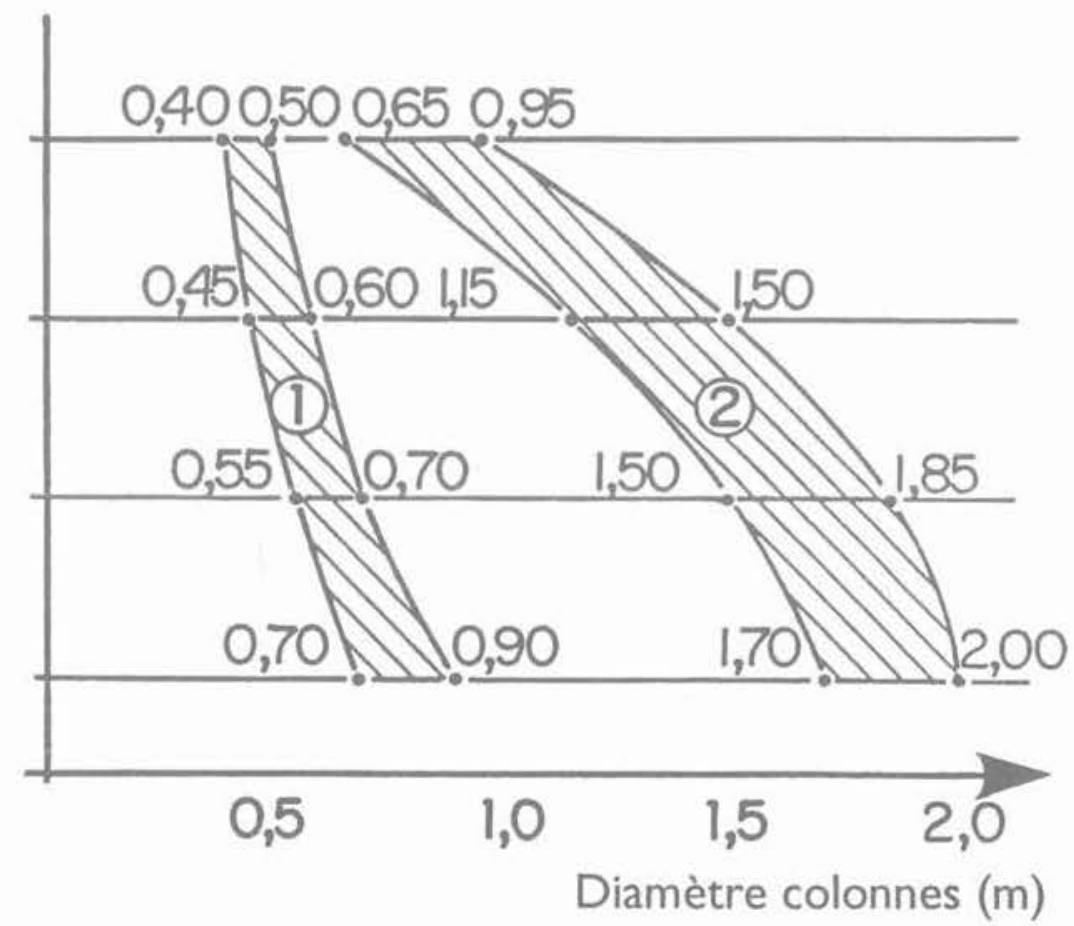

Fig. 3. - Diamètres habituels des colonnes obtenues dans différents terrains.

Fig. 3. - Typical diameter of columns obtained for different ground conditions. 

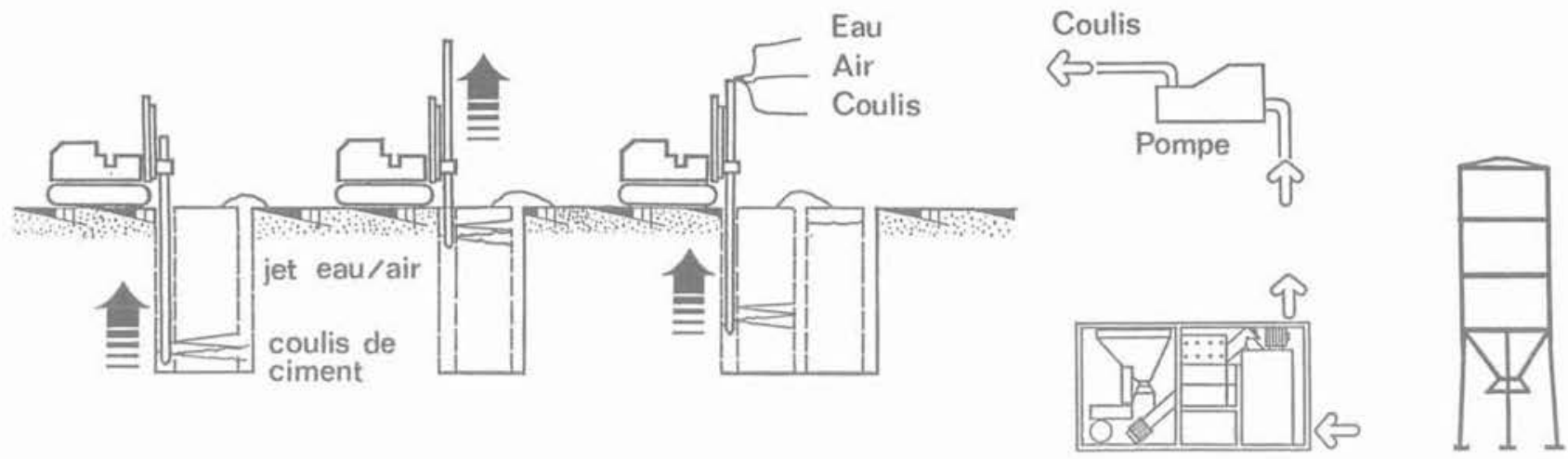

Ciment

Fig. 4. - Jet Mix, réalisation de panneaux.

Fig. 4. - Jet Mix, construction of panels.

- le préjetting : on désagrège une partie du terrain par un jet d'eau à haute pression (avec ou sans air annulaire) avant de réaliser le jet proprement dit. L'intérêt de cette méthode est généralement d'éviter un gaspillage très important de matériaux nobles dans le spoil lorsque le terrain et les performances demandées requièrent de très fortes énergies de jetting, avec des économies supérieures au coût occasionné par l'exécution des colonnes en deux phases. Le préjetting est utilisé aussi parfois pour élargir le forage et faciliter la sortie du spoil vers la surface.

\section{LES DOMAINES D'APPLICATION DU JET GROUTING}

\subsection{Terrains meubles}

non ou difficilement injectables

Un premier domaine type d'application est celui du traitement des terrains meubles non ou difficilement injectables tels que les limons et argiles, que l'on cherche généralement à consolider, quelquefois à étancher (cas des limons et sables très fins).

\subsubsection{Traitement de masse}

Citons comme cas types d'application :

- consolidation des terrains pour l'accostage ou le démarrage de tunneliers, exemple de la ligne D du métro de Lyon au départ du puits Cavenne : un traitement de consolidation de masse des limons et alluvions du Rhône a été réalisé à partir d'un maillage de $1 \mathrm{~m}$ de colonnes de diamètres $1,2 \mathrm{~m}$ (fig. 5) ; ce traitement a été exécuté à travers une forêt d'anciens pieux de bois et limité à environ $2 \mathrm{MPa}$ de résistance mécanique pour ne pas gêner le travail ultérieur du tunnelier :

- consolidation des terrains pour le creusement de galeries en terrain mou, exemple du lot 108 du métro de Singapour (fig. 6) : les argiles et vases molles de SPT inférieur à 2 ont été consolidées sur une couronne de $1,5 \mathrm{~m}$ d'épaisseur autour de deux futurs tunnels superposés de $5,9 \mathrm{~m}$ de diamètre à des profondeurs

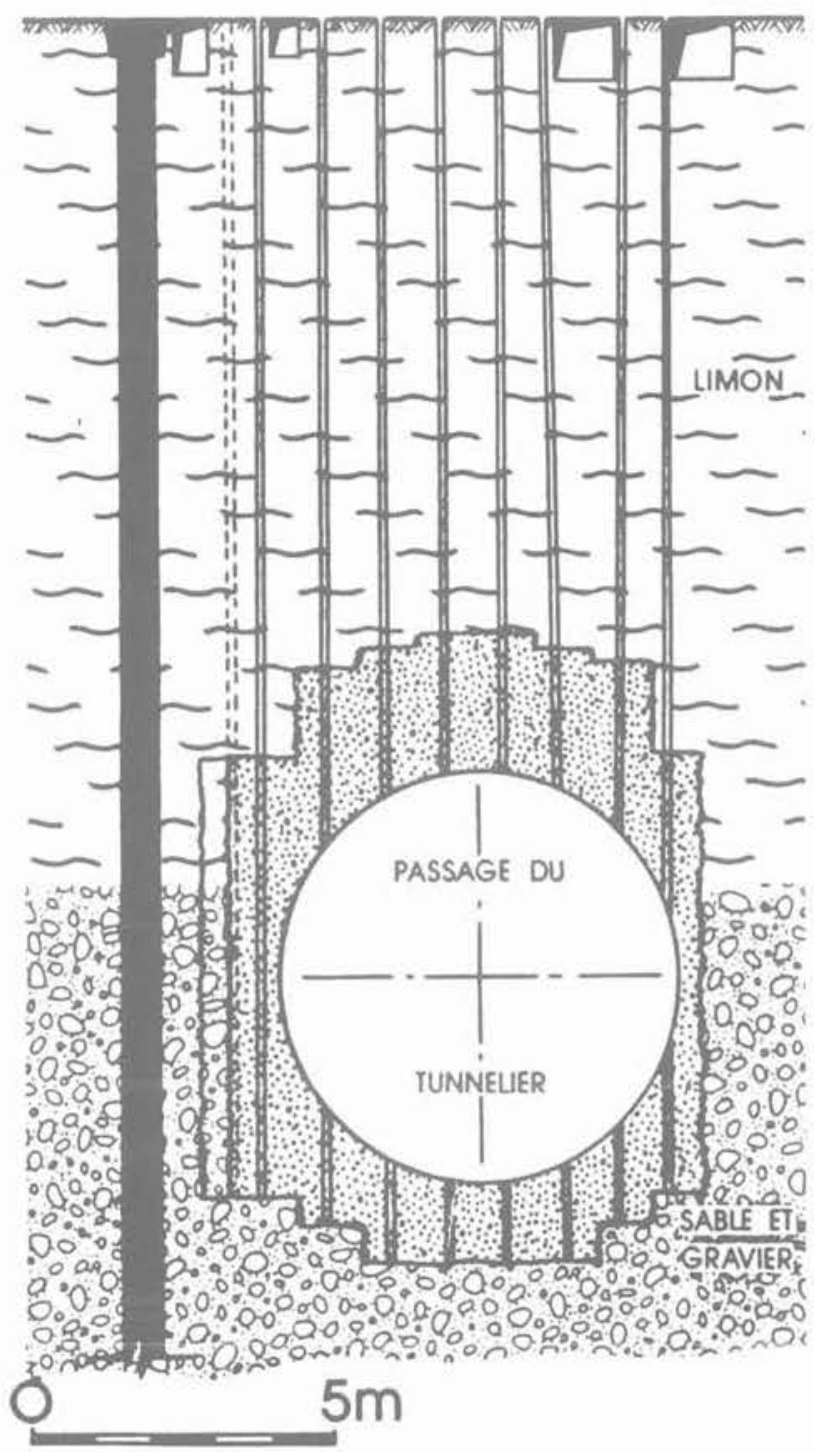

Fig. 5. - Métro de Lyon, traitement de sortie du puits Cavenne. Fig. 5. - Lyons Metro, treatment of Cavenne shaft 


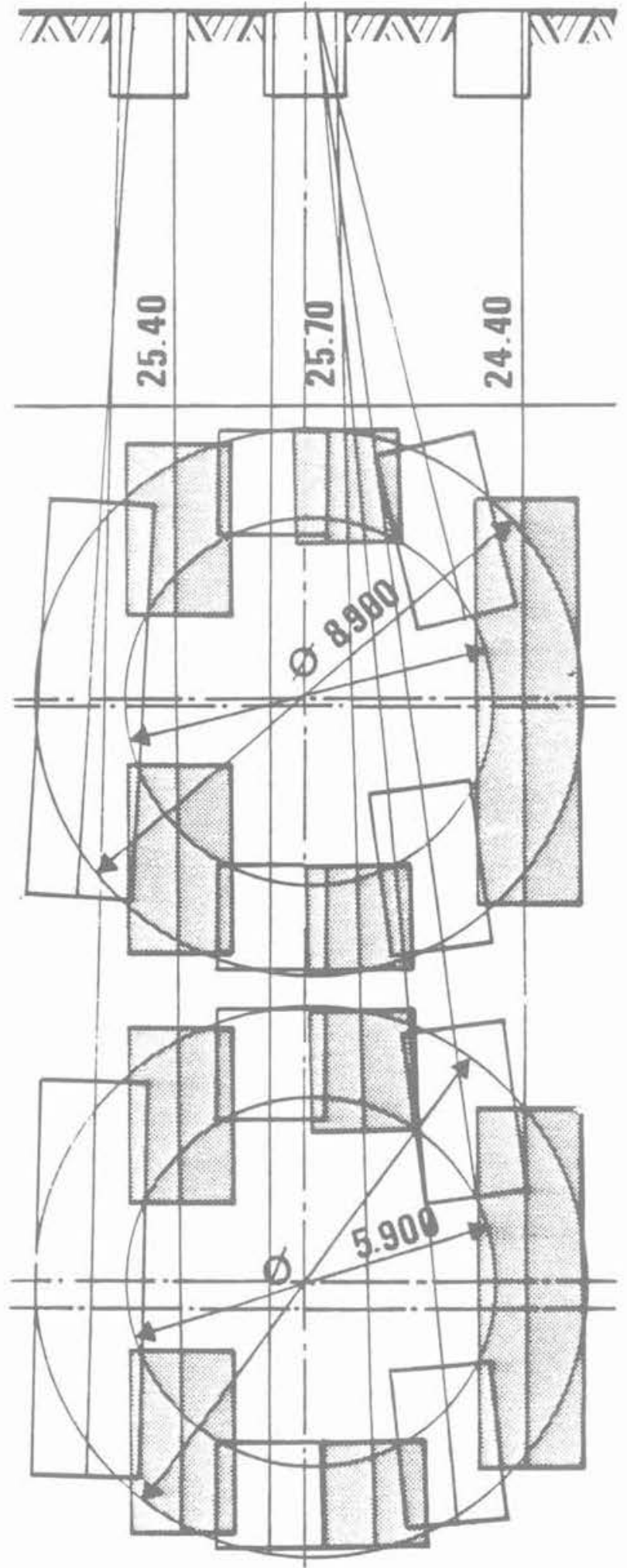

Fig. 6. - Métro de Singapour, lot 108, traitement annulaire des tunnels superposés. Fig. 6. - Singapore Metro. MRT 108, annular treatment for superimposed tunnels.

atteignant $25 \mathrm{~m}$ par un réseau de colonnes sécantes de $1.8 \mathrm{~m}$ de diamètre réalisées depuis la surface.

\subsubsection{Traitements linéaires}

Citons comme cas types d'application :

- coupure de couches épaisses d'altération d'un substratum rocheux sous un barrage en terre, exemple du barrage de Thika au Kenya (fig. 7) : la coupure est constituée d'une rangée de colonnes sécantes de $1,2 \mathrm{~m}$ de diamètre et $80 \mathrm{~cm}$ entre axes jusqu'à $35 \mathrm{~m}$ de profondeur dans des argiles limoneuses moyennement compactes avec blocs et bancs rocheux, prolongée par un voile injecté monolinéaire dans le rocher jusqu'à $70 \mathrm{~m}$; les foreuses de jet sont installées sur des plateformes montées sur rails et déplacées par des treuils suivant une pente moyenne de $20^{\circ}$ le long des rives ;

- voûte parapluie en galerie réalisée à l'avancement. exemple du tunnel Malenchini sur l'autoroute Livourne-Civitavecchia (fig. 8) : présoutènement de la voute d'un tunnel de $12 \mathrm{~m}$ d'ouverture dans des sables limoneux par une couronne de colonnes subhorizontales de jet simple, diamètre $60 \mathrm{~cm}$, longueur 13 à $14 \mathrm{~m}$, armées de tubes de $89 \mathrm{~mm}$ de diamètre et $10 \mathrm{~mm}$ d'épaisseur, 80 bonds consolidés et terrassés en alternance, avec pose des cintres et béton projeté ;

- enceinte autour de puits rectangulaires ou circulaires, exemple du puits 11 de l'émissaire SèvresAchères (fig. 9) : couronne de colonnes sécantes verticales de diamètre $1,2 \mathrm{~m}$ et profondeur $22 \mathrm{~m}$ à travers des limons, sables et graviers et argiles plastiques autour d'un puits de $3 \mathrm{~m}$ de diamètre excavé manuellement.

\subsubsection{Inclusions}

Citons comme cas types d'application :

- fondations de radiers sur terrains compressibles, exemple de la prison de Lonay en Suisse (fig. 10) : radier fondé sur des colonnes de diamètres variables entre 60 et $115 \mathrm{~cm}$, de capacité portante 40 à $120 \mathrm{t}$, à travers $25 \mathrm{~m}$ de limons de cohésion 15 à $30 \mathrm{kPa}$ : - fondations de poteaux isolés sur sols compressibles, exemple de la salle des sports de Servas (fig. 11) : reprise en sous-ceuvre d'un bâtiment fondé sur des limons argileux et ayant tassé par des colonnes inclinées de diamètre $130 \mathrm{~cm}$ et 5 à $6 \mathrm{~m}$ de profondeur ;

- fondations d'ouvrages en présence de poches d'argile karstique, exemple du tunnel de Saint-Germainde-Joux sur l'autoroute A 40 dans le Jura (fig. 12) : consolidation des argiles molles (pression limite $0.2 \mathrm{MPa}$ ) rencontrées dans la partie inférieure du tunnel pour appuyer les cintres de la demi-section supérieure du tunnel déjà terrassée et la voûte inverse de la demi-section inférieure.

\subsection{Terrains meubles injectables}

La recherche d'une résistance mécanique élevée. l'existence d'une couverture faible, l'interdiction d'emploi de produits chimiques, ou plus simplement l'économie du projet peuvent conduire à l'utilisation des procédés de jet pour la consolidation et l'étanchement de terrains meubles injectables par ailleurs sans problèmes, tels que les sables et graviers. 


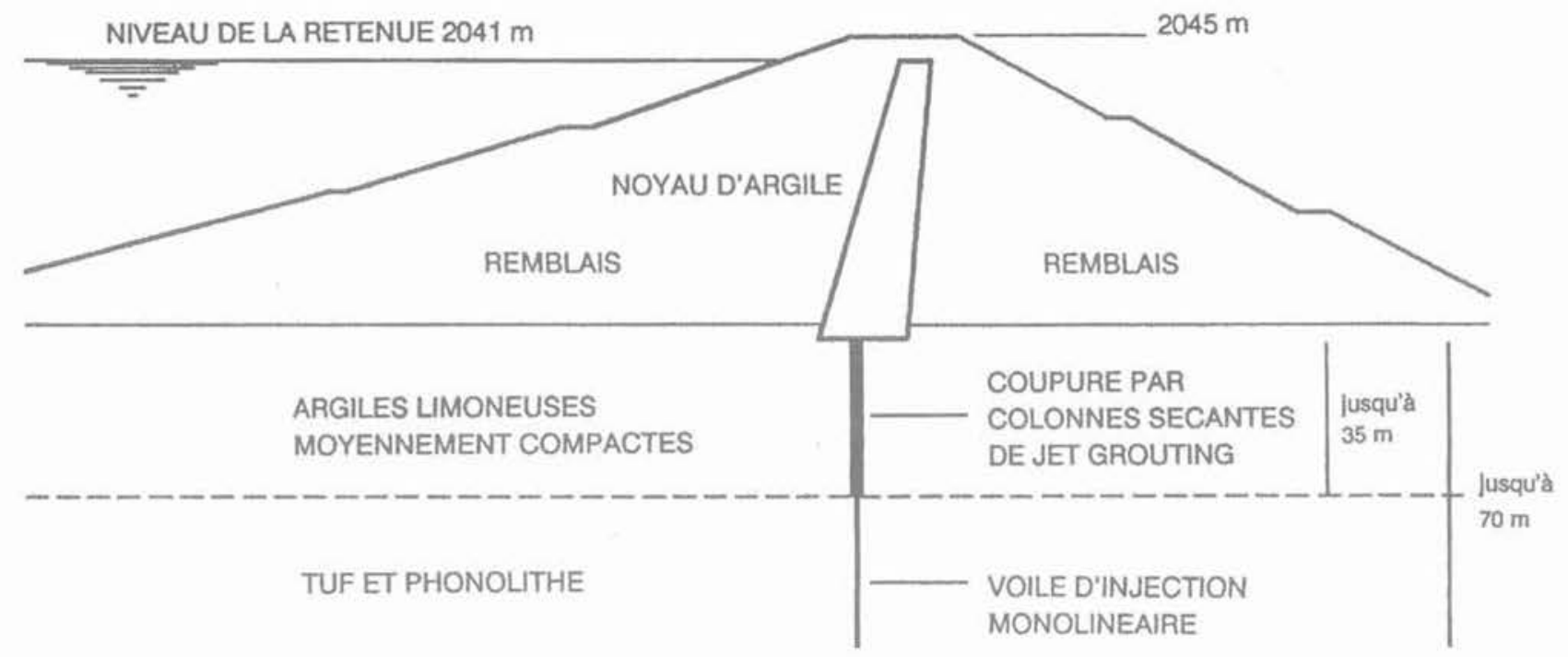

Fig. 7. - Coupe transversale de la coupure étanche du barrage de Thika. Fig. 7 - Cross section of the Thika Dam, Kenya.

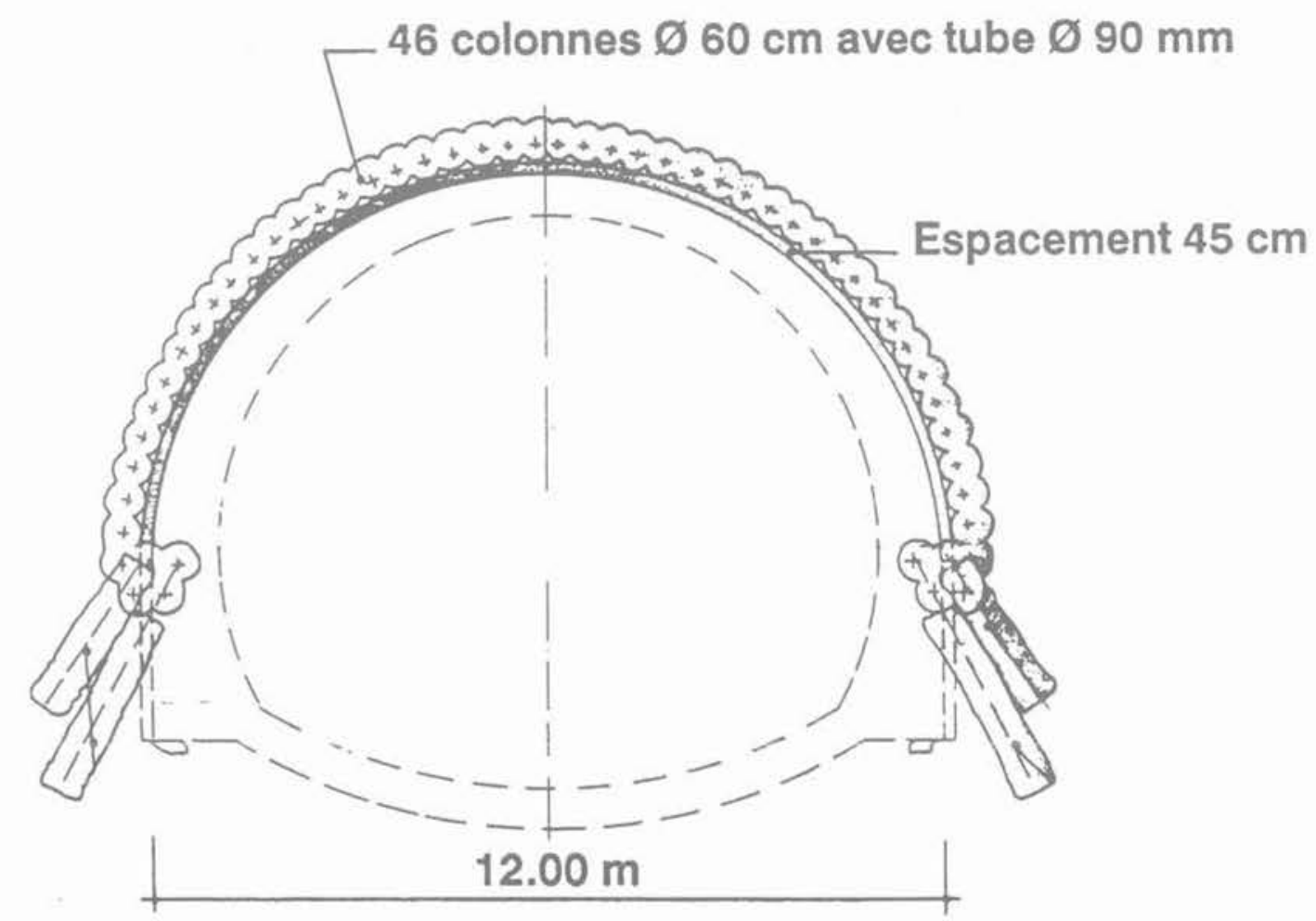

Fig. 8. - Tunnel Malenchini, Jet horizontal.

Fig. 8. - Malenchini tunnel, horizontal jet grouting. 


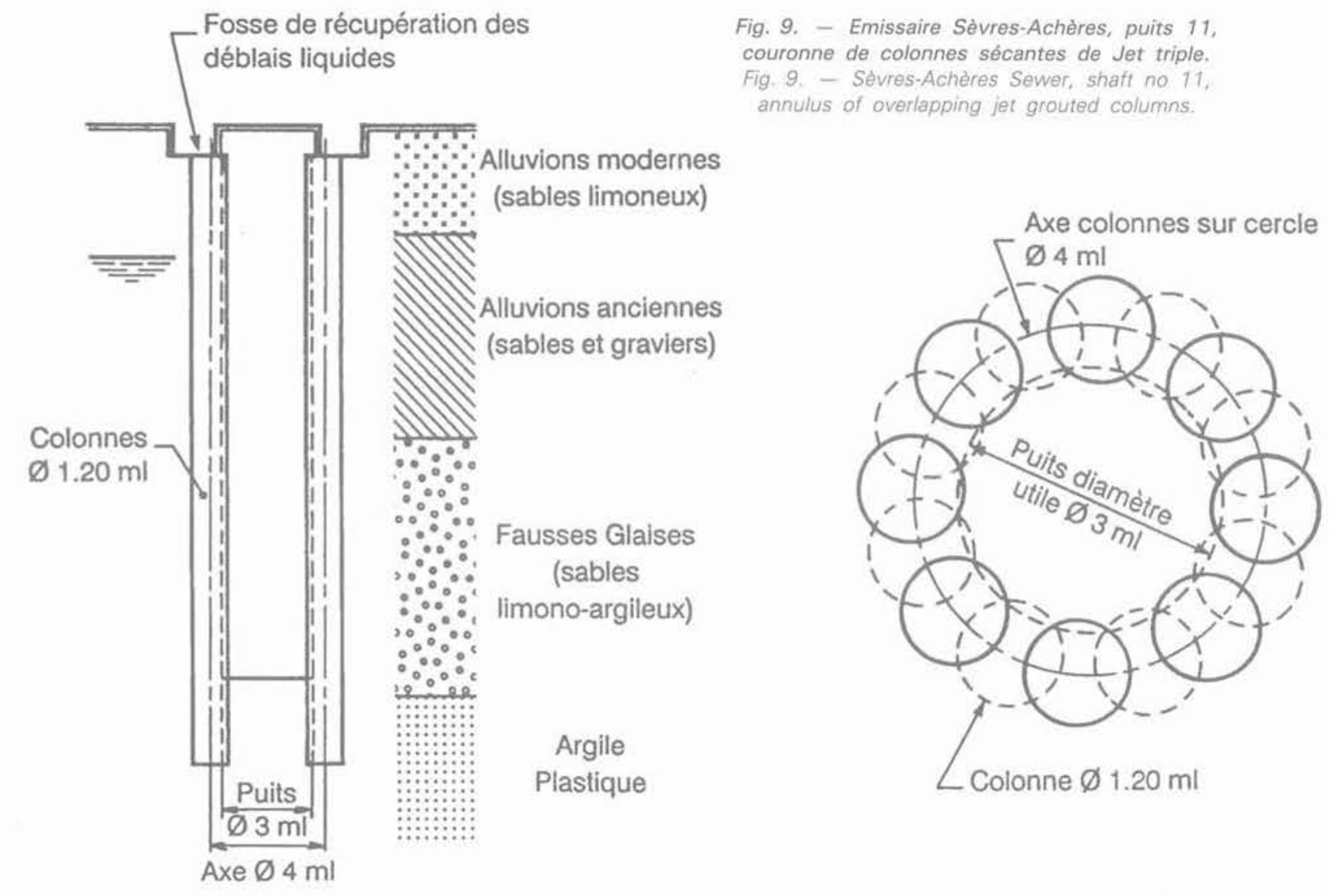

Fig. 9. - Emissaire Sèvres-Achères, puits 11, couronne de colonnes sécantes de Jet triple, Fig. 9. - Sèves-Achères Sewer, shaft no 11. annulus of overlapping jet grouted columns.
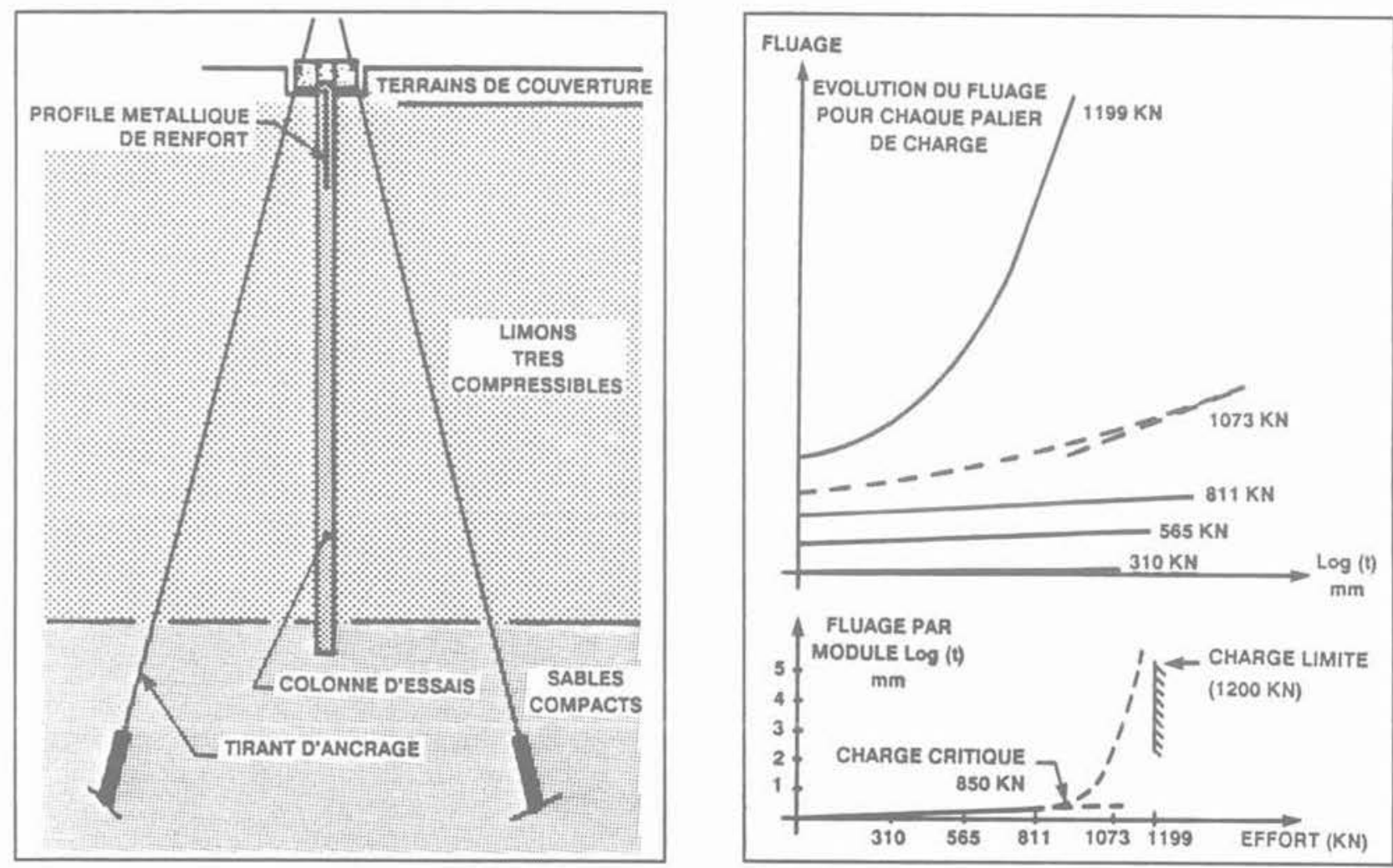

Fig. 10. - Lonay: essai de charge sur colonne.

Fig. 10. - Lonay, load test on a column. 


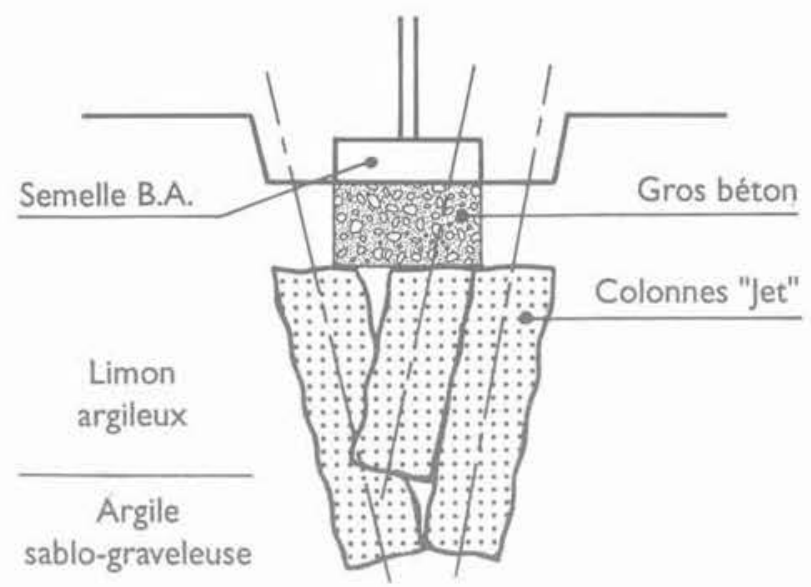

Fig. 11. - Servas, reprise en sous-ceuvre d'un poteau isolé. Fig. 11. - Servas, underpinning of an isolated column.

\subsubsection{Traitement de masse}

Citons comme cas types d'application :

- constitution de radiers d'étanchéité, éventuellement en forme de voûte inversée appuyée sur des parois latérales, exemple de la fouille du complexe Leonardo da Vinci à Lecco (fig. 13) : réalisation d'un réseau de colonnes sécantes de diamètre $1,5 \mathrm{~m}$ jusqu'à $20 \mathrm{~m}$ de profondeur dans des sables fins à grossiers ;

- constitution de massifs poids sous les bâtiments mitoyens d'une fouille, exemple de la poste de Cologne (fig. 14): formation d'un mur de $8 \mathrm{~m}$ de hauteur et $20 \mathrm{MPa}$ de résistance reprenant en sous-œuvre les fondations existantes, par une combinaison de quatre colonnes d'inclinaison et longueur différentes, dans les alluvions du Rhin.

\subsubsection{Traitements linéaires}

Citons comme cas types d'application :

- réalisation de voiles d'étanchéité en profondeur. au moyen de colonnes sécantes, de panneaux ou de combinaisons de colonnes et de panneaux, exemple du tunnel Willemspoort à Rotterdam (fig. 15) : réalisation entre 17 et $38 \mathrm{~m}$ de profondeur avec une glissière de $40 \mathrm{~m}$ montée sur grue, de colonnes verticales de 120 et $170 \mathrm{~cm}$ de diamètre reliées par des panneaux de jet, en prolongation d'un mur composite de tubes et de palplanches;

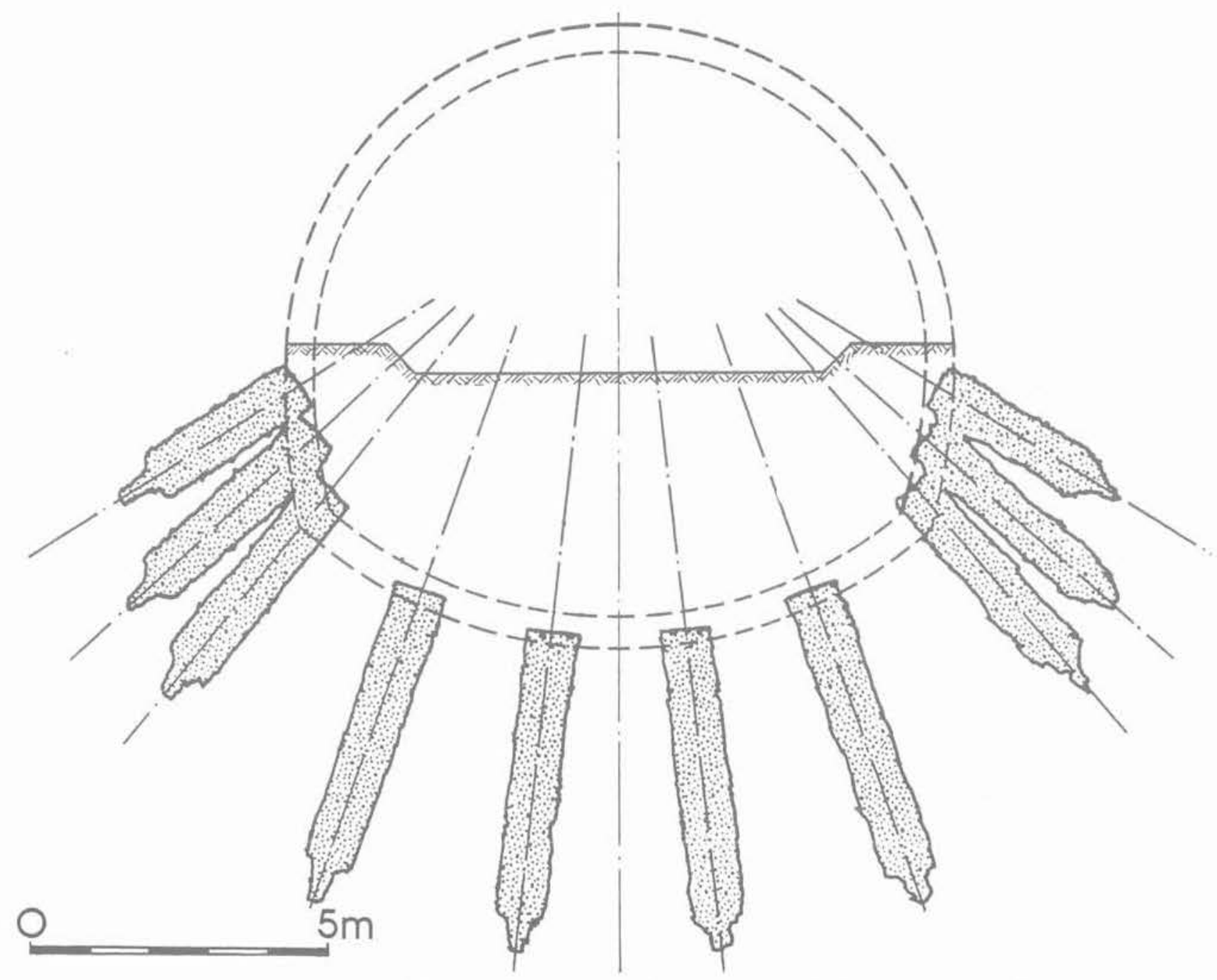

Fig. 12. - Tunnel de Saint-Germain-de-Joux, traitement des appuis de cintre et du radier. Fig. 12. - Saint-Germain-de-Joux tunnel, treatment of base slab and arch support. 

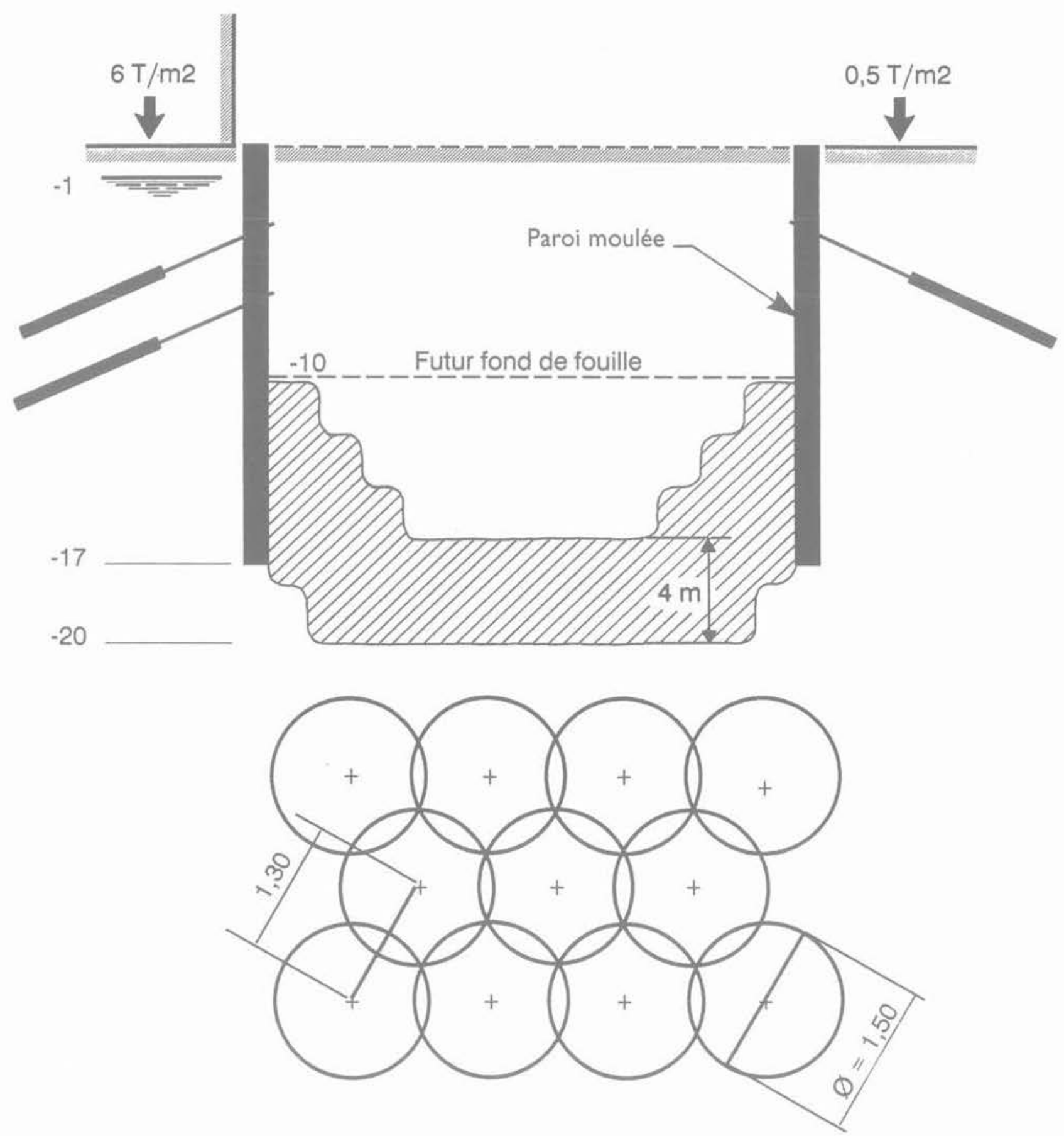

Fig. 13. - Lecco, fond étanche en colonnes sécantes de Jet double.

Fig. 13 , Lecco, watertight arch blanket using double jet overlapping columns.

- réalisation de murs de type berlinois, exemple du collecteur Sesto San Giovanni à Milan (fig. 16) : poteaux constitués de colonnes armées, diamètre $80 \mathrm{~cm}$, espacement $1 \mathrm{~m}$, de part et d'autre d'un collecteur construit en tranchée couverte dans des sables limoneux.

\subsubsection{Inclusions}

Citons comme cas types d'application :

- consolidation par effet de groupe d'inclusions de jet, exemple de la restauration de la Cour Carrée du musée du Louvre (fig. 17) : renforcement des allu- vions de la Seine par une double rangée de colonnes en quinconce de diamètre $80 \mathrm{~cm}$ espacées de $120 \mathrm{~cm}$ au contact de vestiges archéologiques (murs d'anciennes douves) pour fonder une dalle de couverture et reprendre des poussées latérales;

- protection d'immeubles contre les tassements provoqués par des excavations profondes, exemple du métro de Caracas (fig. 18) : en face des immeubles sensibles, il a été nécessaire de réaliser des colonnes de jet inclinées depuis la surface pour réduire les tassements lors du passage d'un bouclier à pression des terres. 


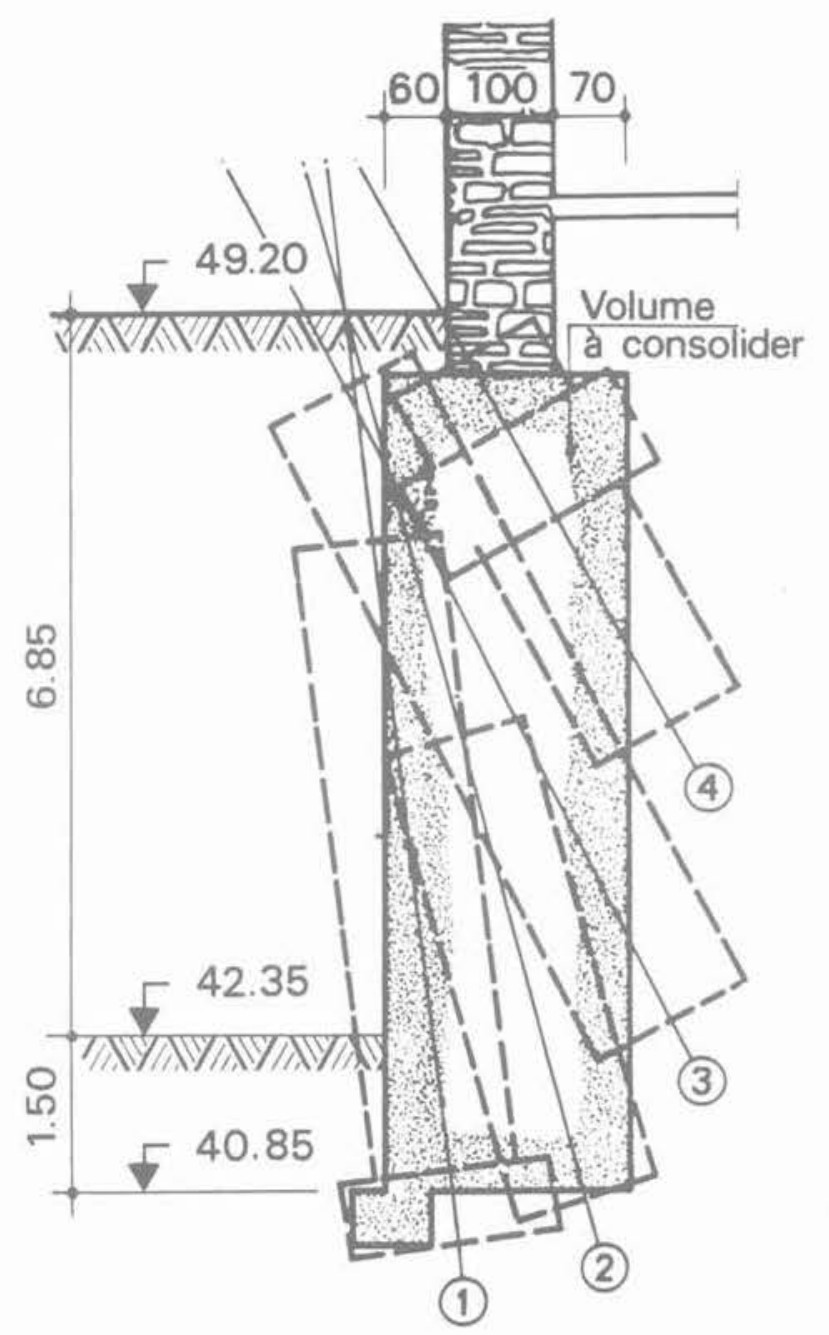

Fig. 14. - Reprise en sous-cuuve des batiments de l'ancienne poste à Cologne.

Fig. 14. - Underpinning of the old post office in Cologne prior to adjacent deep excavation.
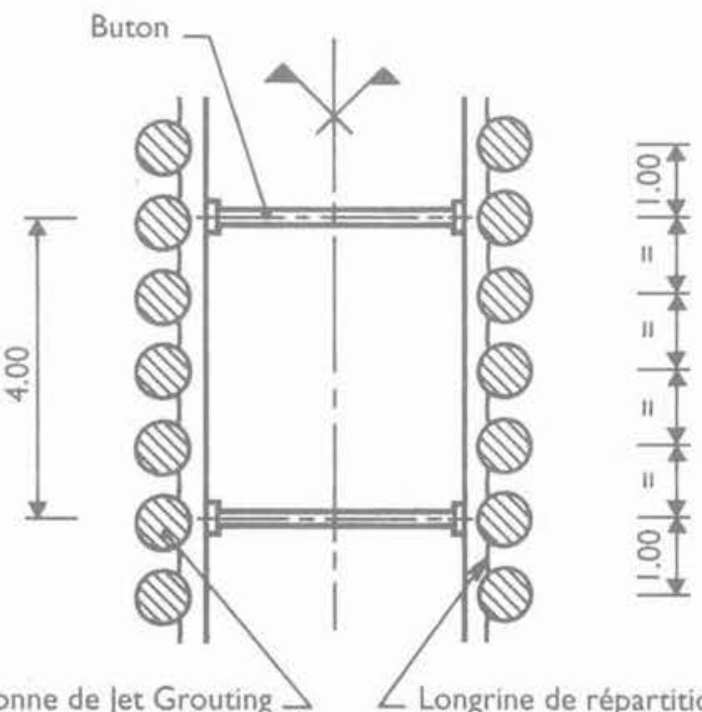

Colonne de jet Grouting avec tube de $73 \mathrm{~mm}$ en béton armé

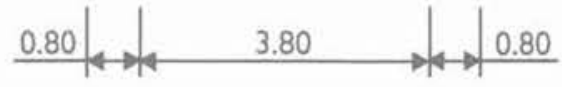

Fig. 16. - Collecteur Sesto San Giovanni, mur de type berlinois en colonnes armées de Jet simple. Fig. 16. - Sesto San Giovanni, main sewer, Berlin wall with single jet columns reinforced with steel tubes.

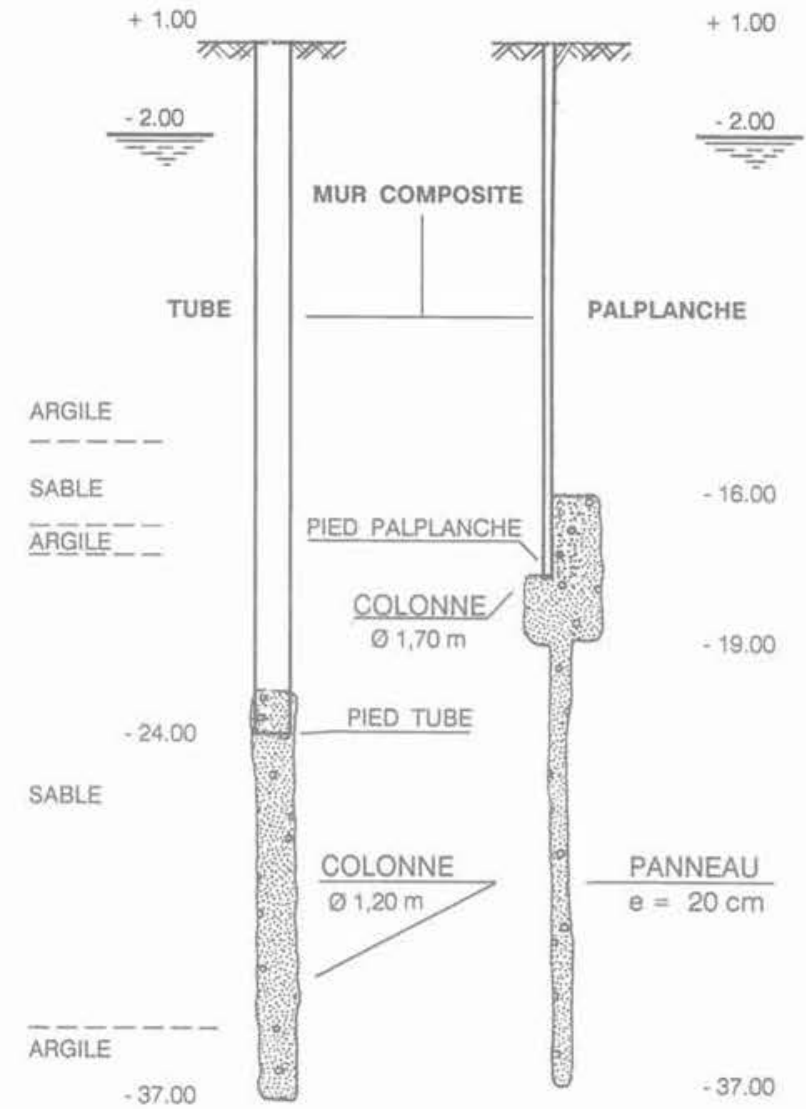

Fig. 15. - Rotterdam, tunnel Willemsspoort, combinaison de colonnes et de panneaux.

Fig. 15. - Rotterdam. Willemsspoort tunnel, combination of columns and panels.

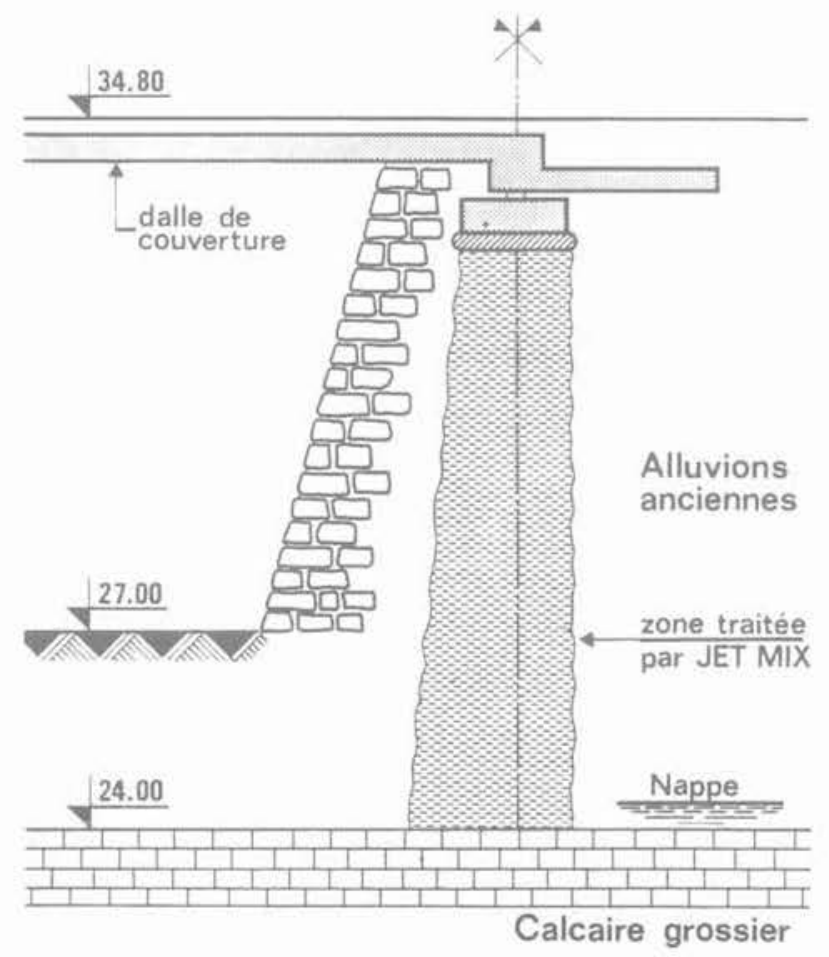

Fig. 17. - Consolidation des terrains d'assise de la dalle de couverture de la Cour Carrée du musée du Louvre. Fig. 17. - Consolidation of the ground supporting the roof of the "Cour Carrée" at the Louvre Museum. 


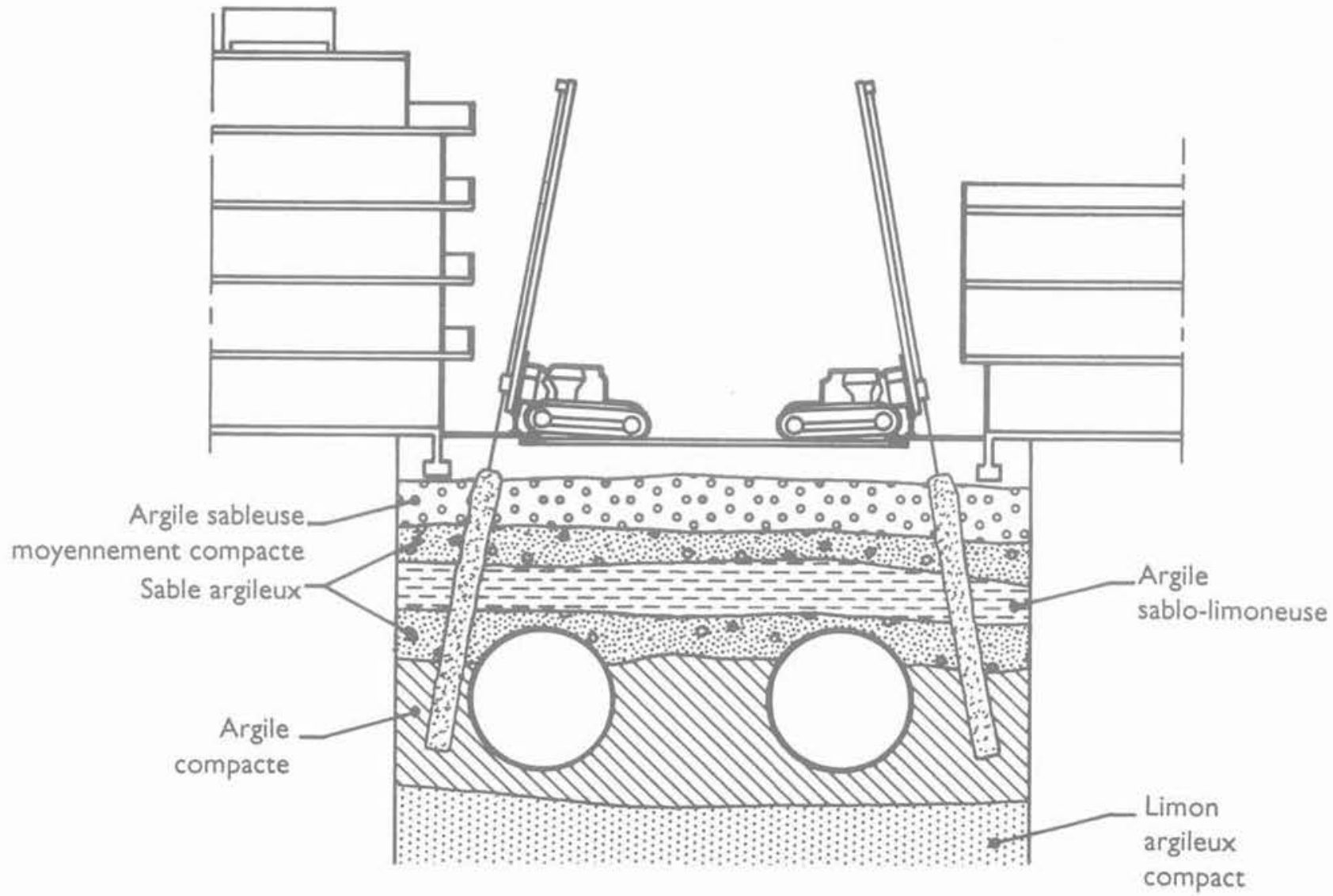

Fig. 18. - Métro de Caracas, protection d'immeubles au droit du passage de tunneliers.

Fig. 18. - Caracas Metro, protection of the buildings along the route of the tunneling machines.

\subsection{Applications particulières}

Le fait que les procédés de jetmix soient mis en ceuvre à partir de forages en petit diamètre leur confère une grande souplesse d'utilisation, en particulier pour la fermeture de fenêtres localisées dans des rideaux de palplanches, des murs en pieux sécants ou en paroi moulée (passage d'une canalisation. dégrafage de palplanches, joints défaillants, etc.) et pour réaliser des protections d'ouvrages depuis la surface en présence d'obstacles enterrés.

Un exemple remarquable de ce type d'application est celui du voile d'étanchéité du barrage de Seujet à Genève dans la partie située sous le radier de l'ancienne usine des forces motrices. Le voile est constitué d'une rangée de colonnes inclinées sécantes de $110 \mathrm{~cm}$ de diamètre et 5 à $13 \mathrm{~m}$ de profondeur. venant au contact du radier fragile des anciens bâtiments rénovés en musée, à travers des alluvions compactes grossières et des moraines (fig. 19). La réalisation des colonnes, en contre pression de la nappe du Rhône, a été effectuée pour moitié depuis l'intérieur de l'usine en activité et le reste depuis un ponton sur le Rhône.

\section{PROBLÈMES POSÉS PAR LES TECHNIQUES DE JET}

\subsection{Définition géométrique et mécanique du résultat}

Le diamètre de la colonne (ou la longueur du panneau), autrement dit le rayon d'action du jet, dépend de la méthode, des paramètres et du terrain. La notion géométrique de cylindre régulier de sol traité est une simplification commode, mais rarement accessible dans la pratique. Il est donc nécessaire de prendre en compte au stade du projet les irrégularités géométriques inévitables induites par le terrain (en particulier pour les rideaux et fonds étanches) et au stade de la réalisation de limiter les variations des paramètres par une mise en œuvre aussi rigoureuse que possible (systèmes automatiques de réglage des vitesses de translation et de rotation des tiges, mesure et enregistrement des pressions et débits).

La précision de l'implantation et de l'alignement du forage à partir duquel est réalisé le jet constitue un autre élément important d'écart entre la théorie et la 


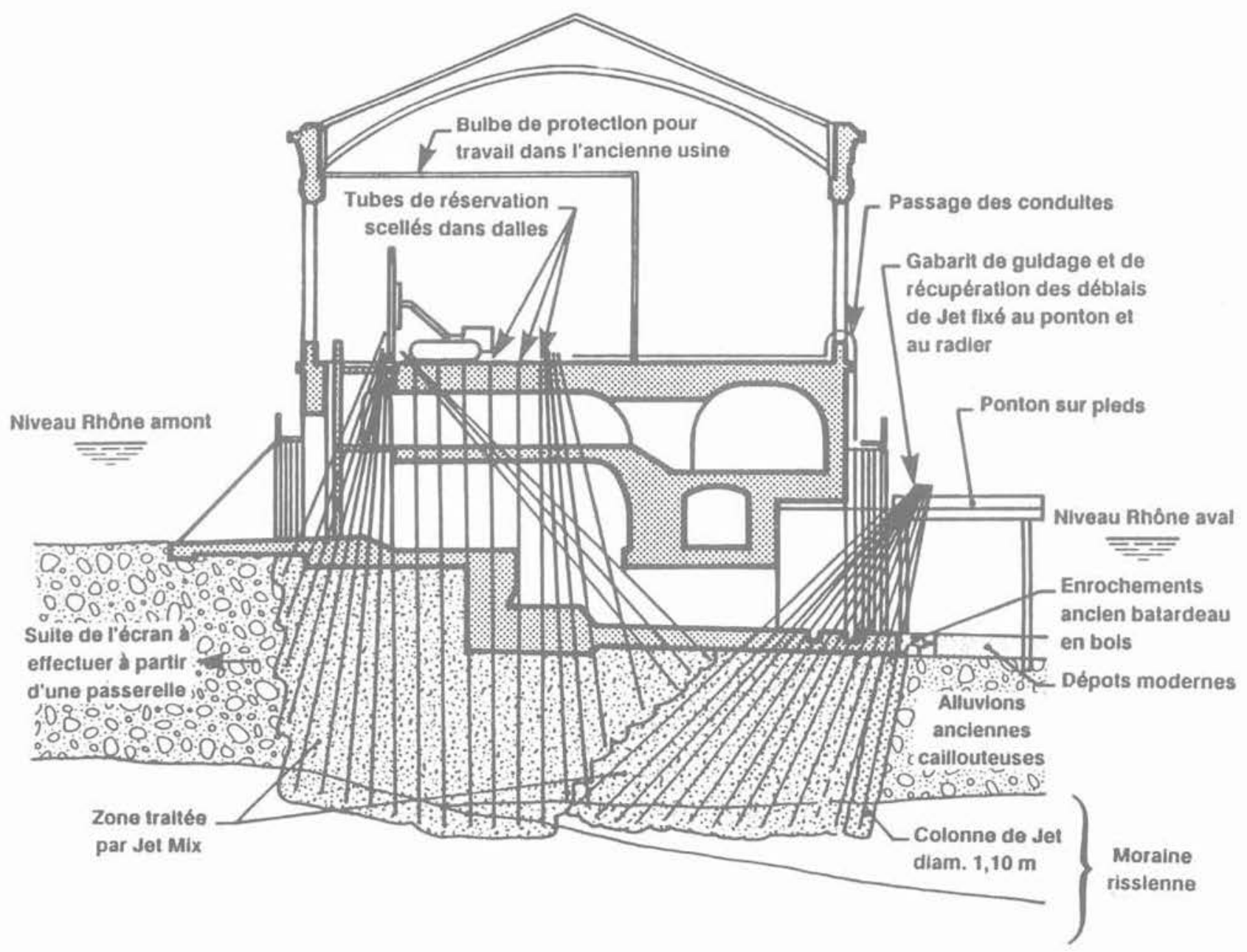

Fig. 19. - Voile d'étanchéité et le long du bâtiment des forces motrices, barrage du Seujet, Genève.

Fig. 19. - Watertight curtain along the length of the pumping station building. Seujet dam. Geneva.

pratique dans la définition géométrique du traitement. Là aussi, il est indispensable de prendre en compte des tolérances de déviation dès le stade du projet et. selon le but recherché, de mettre en œuure des méthodes et matériels adaptés. Cela peut aller (comme par exemple pour le rideau de colonnes sécantes du barrage de Thika) jusqu'à la réalisation de préforages les plus soignés possibles (inclinomètres sur le mât des machines, tiges lourdes autoguidées, mesure de la position de chaque forage par inclinométrie avant traitement).

La résistance mécanique du * béton de sol " obtenu dépend du terrain en place (en plus des paramètres du traitement y compris le dosage du coulis). S'il est relativement facile d'obtenir des résistances importantes en utilisant un coulis fortement dosé en ciment. on cherche rarement des valeurs supérieures à $5 \mathrm{MPa}$ (en compression simple) pour des raisons économiques. Lorsqu'au contraire, les caractéristiques mécaniques du matériau doivent être maintenues dans les valeurs faibles, cette maîtrise est délicate à obtenir. On doit faire appel à des coulis stables bentonite ciment peu dosés en ciment (comme par exemple dans le cas du métro de Lyon) et même rechercher à substi- tuer un maximum de terrain par le coulis dont on connaît les caractéristiques (sur le barrage de Thika, un préjetting à l'eau a permis d'enlever la majeure partie du terrain, le jetting au coulis effectué dans une phase différée a pu alors se substituer au mélange eau-terrain, ce qui n'est pas le cas en jet triple classique où le coulis se mélange à l'eau et au terrain et ressort en surface dès qu'on commence la colonne).

\subsection{Sujétions d'exécution}

Pendant le traitement, le terrain environnant non atteint par le jet n'est normalement pas affecté, hormis une élévation temporaire des pressions interstitielles. Mais si pour une raison quelconque il y a blocage du spoil dans l'espace annulaire entre tiges et forage, on a rapidement claquage du terrain et risque de soulèvement important et brutal. Il est donc nécessaire de surveiller la libre remontée du spoil pendant la mise en ceuvre et d'arrêter rapidement le jet si une anomalie apparaît. Quelquefois, il faut tuber en gros diamètre (environ $150 \mathrm{~mm}$ ) la partie supérieure du forage ayant tendance à s'ébouler (comme par exemple dans les argiles très molles du lot 108 
du métro de Singapour, où le prétubage associé à une exécution des colonnes en deux tranches descendantes a résolu le problème des soulèvements qui au départ atteignaient jusqu'à $50 \mathrm{~cm}$...).

Le volume du spoil à évacuer est théoriquement égal aux volumes d'eau et de coulis pompés (dans les terrains saturés). Cela peut constituer une sujétion très importante sur les chantiers où la réalisation de tranchées et bacs de récupération de grand volume pose problème (rues étroites, trottoirs, caves, galeries, etc.). Parfois il est nécessaire de pomper le spoil à la sortie même du forage pour ne pas polluer (comme par exemple pour les colonnes du barrage de Seujet faites sur ponton, pour lesquelles tout écoulement du spoil chargé en ciment vers les eaux du Rhône était interdit)

Dans les sables grossiers et les graviers, le mélange grains du terrain-ciment frais n'est pas stable et décante avant la prise du coulis. De plus, les sables et graviers forment un milieu filtrant qui provoque un essorage très important du coulis à travers la surface de contact colonne-terrain, surtout s'ils ne sont pas saturés. La conséquence est une baisse de niveau rapide du mortier de jetmix, qu'il convient de compenser avant la prise du coulis pour éviter une coupure de la colonne. Cette précaution est particulièrement nécessaire pour les chantiers de reprise en sousœuvre (il suffit généralement de prévoir une prétranchée formant un réservoir reliant entre elles les têtes de colonnes).

\section{CONTRÔLES}

Les essais et contrôles doivent permettre d'apprécier la géométrie et la qualité du traitement effectivement réalisé. On peut distinguer:

- colonnes d'essais déterrées : celles-ci permettront d'étalonner les paramètres en fonction du terrain et du résultat voulu. Si le déterrement n'est pas possible, on cherchera les résultats d'une autre façon (carottages, forages recoupants, essais de charge, etc.), mais les tolérances du projet devront considérer un degré d'incertitude plus élevé:
- contrôle du spoil : la densité du spoil est intermédiaire entre celle du terrain en place et celle des fluides envoyés ; son suivi donne une idée du volume de terrain remanié (plus la densité du spoil est proche de celle du terrain, plus le volume de matériaux mélangés est important, et par conséquent le diamètre), mais souvent avec une grosse incertitude due à une connaissance imparfaite de la densité du terrain naturel. Le spoil est un échantillon frais du mélange de jetmix, lequel différera en place par décantation et essorage, donc enrichissement en ciment avant prise du coulis. Mesurer les caractéristiques mécaniques du spoil permettra donc d'apprécier par défaut celles du mélange final (ce qu'on aura pu étalonner à l'occasion des colonnes d'essai) ;

- forages de contrôle : des sondages carottés pour des colonnes de forte résistance, des essais pénétrométriques pour des colonnes de faible résistance permettent de contrôler les caractéristiques mécaniques du traitement. De façon plus incertaine, on peut apprécier la géométrie obtenue par des forages recoupants (sondages inclinés dans le plan du voile du barrage de Thika par exemple). On peut également juger globalement le résultat (essai de pompage avant terrassement pour un fond étanche par exemple). Mais la difficulté sera souvent de déterminer l'implantation de points complémentaires de traitement en cas de défaut constaté

\section{CONCLUSION}

Les techniques de jet grouting viennent compléter remarquablement les moyens dont dispose le spécialiste pour l'amélioration des sols. Leur souplesse d'exécution permet leur adaptation à de nombreux cas en frontière des techniques habituelles d'injection et de fondation.

Les quelques lignes directrices et exemples d'application cités dans cet article pourront peut-être contribuer à en préciser les limites d'utilisation et à faciliter leur développement dans la construction. 